\title{
SUCCESS AND FAILURE REVISITED IN THE IMPLEMENTATION OF NEW TECHNOLOGY: SOME REFLECTIONS ON THE CAPELLA PROJECT
}

\author{
Tom McMaster ${ }^{1}$ and David Wastell ${ }^{2}$ \\ ${ }^{I}$ Information Systems Institute, University of Salford, UK; ${ }^{2}$ Department of Computation, \\ UMIST, Manchester, UK
}

\begin{abstract}
Reports about success and failure in IS implementation initiatives are plentiful, but the use of such labels is too often uncritical, pejorative and simplistic. We reflect on a two-year implementation project that had elements of both failure and success, using this to revisit and examine more closely the meanings of the terms, and to consider other key dimensions of the innovation process, such as the pivotal role of organizational culture and crises in decisively shaping outcomes.
\end{abstract}

\section{INTRODUCTION}

In mid-July 2003, 250 British Airways check-in and customer service staff staged a two-day wildcat strike at London's Heathrow Airport in protest at the company's unilateral decision to implement a new swipe-card time-recording system. Although the proposal for such a technological innovation undoubtedly looked extremely attractive on paper, the upshot was that around 80,000 passengers suffered massive inconvenience by the action, and it cost the company in the region of $£ 50$ million, not to mention the incalculable damage to its image and passenger relations according to subsequent and extensive media coverage. By the end of that month the plan had been shelved if not entirely abandoned, thus demonstrating once again that a long tradition of innovation failure - especially when it comes to the implementation of new technology - is a phenomenon that appears no less a 
problem today, even in the largest and most recognisable of branded companies, than it has been in the past with others.

It is generally considered that the failure to implement innovative technical systems, for whatever reason, is synonymous with the term 'system failure' (see for example Lyytinen and Hirschheim, 1987). We suggest that the designation of such judgemental and politically loaded terms as failure and success merely reflects a single view. In the above case, and perhaps more often than not, it tends to reflect a managerial perspective that may be prone to 'pro-innovation bias', that is, the assumption that the innovation is intrinsically good and desirable, while subjugating or denying the views of those who see it as an imposition and a threat. Nevertheless, alternative views may be at least as valid and compelling as those promoted by managers. The strikers at BA might well consider the system to have been a complete success, since its trajectory seems to have ensured that so-called 'spanish practices'1 among themselves and other staff are likely to remain unchallenged for the foreseeable future. It would seem that pinning the label of success or failure to a project sometimes depends on which side one finds oneself politically aligned.

The purpose of this paper is not merely to highlight the fact that those with different perspectives will interpret the implementation of the same technical innovation in very different ways, important though this is. We wish equally to draw attention to the troublesome nature of the oversimplified one-dimensional concept that is frequently signified by the labels 'failure' and 'success' whenever they are used to describe the introduction of new technical systems into organisational arenas. Too often, these terms are employed to describe the object of implementation efforts, when closer examination would instead reveal far less certainty, much wider ambiguity, and immensely greater complexity surrounding the implementation of new technology, where the product of these efforts is more likely to be a whole range of benefits and disbenefits. Some of these are no doubt predictable, but others may not be. We feel therefore that a more critical approach toward these terms and concepts is more likely to provide richer explanations about why there are often very different perceptions about the same processes, the elusive nature of success and failure, and the peculiar fact that what is often considered failure today, may not be considered so tomorrow (Bignell \& Fortune, 1987, Larsen \& Myers, 2000).

${ }^{1}$ A term in common currency in press reports of the time to denote unofficial rule 'bending' in this case, leaving the job early and having colleagues 'clock-out' for them - as an example of one of the practices that the system was designed to combat. 
Here we recount an attempt to introduce a CASE tool along with a related methodology into the software development department of a large public service organisation that in one sense seems to have failed - that is, the technology failed to infuse or become incorporated into the organisation's working practices, and was finally abandoned. Despite this however, at the end of the 2-year implementation exercise the organization found and felt that it was significantly better off in a number of respects than it was at the start of the project directly as a result of the processes involved, and in ways that were not, and could not have been foreseen. Was this then a failure? Such stories concerning the ephemeral and ambiguous nature of success and failure are not common, but we are sure that while rarely reported, nevertheless the phenomenon itself is far more widespread than it seems.

The work presented here represents the authors' reflections about an EU funded project known as the CAPELLA project, in which they played important roles. The paper is organised in the following manner; in the next section we discuss some issues related to success and failure and its meanings in the context of IS based innovation that may be found in the literature. In section 3, we comment upon the research approach adopted in the course of the CAPELLA project, which we describe in more detail in section 4 . In the final section, we provide a discussion along with some tentative conclusions.

\section{BACKGROUND}

Various works discuss failure both in terms that are generally applicable, and in terms that are specific to the IS and IT research communities. We include the seminal works of Bignell \& Fortune (1984), and Lyytinen \& Hirschheim (1987). The former describe failure as being an assessment of the outcome of an activity based upon the values of the person making the judgement, and we find that failure is not a single thing, but is usually multicausal, with multiple effects. While it remains an elusive and indistinct concept, failure is nonetheless about the evaluation of a performance set against an expectation, and there is a requirement for it to be historically contextualised, since, as suggested above, often what might have once been perceived as a failure, may not be seen as such on a different occasion.

Lyytinen and Hirschheim (1987), who are specifically concerned with the term as it applies to the IS research communities describe four 'classes' of failure, namely, correspondence failure, process failure, interaction failure and expectation failure. Correspondence failure means that design objectives have not been met. Process failure relates to the development 
process where budget, time or other resource allocations have overrun to the point where any benefits expected from the proposed system have now been negated, or where a workable system has not been achieved. Interaction failure is the argument that the low-level use of the system can be interpreted as failure, and expectation failure is simply that the system has failed to meet the expectations of at least one stakeholder group. These authors additionally take the view that the first three classes of failure may be subsumed by expectation failure, since this is effectively a superset of the others.

Sauer (1993) likewise addresses the IS community, providing as he does a number of case studies. His definition differs somewhat from Lyytinen and Hirschheim insofar as he limits his definition of failure only to those systems that are neither under development, nor in operation, eliminating some at least of Lyytinen \& Hirschheim's category of interaction failure. Since the system may be serving at least one person's interest, it therefore cannot be described as a failure. He proposes a 'triangle of dependence' model where the information system depends on the project organisation, the organisation depends on its supporters, and the supporters in turn depend on the information system. The dynamics involved in this tripartite dependency coalition are necessary for understanding system failures, which in short are due to disruption or dissonance in the loop.

Flowers (1996) also provides a number of case studies in IS failure, and his definition of failure generally corresponds to those offered by Lyytinen and Hirschheim, however he proposes a factor-based causal model ('critical failure factors'). These include factors relating to the organisational context, such as hostile culture and poor reporting structures; project management, including over-commitment and political pressures; and conduct of the project, including factors related to various phases of the project that he envisages as a traditional waterfall sequence of activities. Flowers also suggests that most failures result in the players 'burying the facts' of the case, no doubt to avoid embarrassment and fault attribution.

In addition to the above, various individual studies of specific system failures have been reported in the IS research literature. These include Robinson (1994) on the failure of the London Ambulance Service, Kautz and McMaster (1994) on the failure to adopt SSADM in a local government IT department. Wastell (1996) on a similarly abortive attempt to introduce SSADM into the private sector, and McMaster et al (1997) on the failure to adopt a technological innovation into a car-park environment in a UK Institute of Higher Education, to name a few among many others.

Similarly, studies of IS 'success' are not in short supply. These include Teng, et al. (1998), Bussen and Myers (1997), Seddon (1997), and Carr and Johansson (1995) to provide some examples. In addition, the IS literature - 
especially that with a focus on diffusion studies - is not short on examples proposing 'critical success factors' to guide would-be implementers through the hazards of their strange esoteric activities. However, almost as rare as hens' teeth are studies that treat failure / success as the ambiguous and ephemeral entities that we take them to be here.

We conclude this section by observing that studies of successful projects, and studies of unsuccessful projects are relatively plentiful in the IS research literature, while studies where the focus is not on the project but on the nature of success and failure per se, are far less so, and it is upon this that we focus our attention. Before moving to a description of our case, we offer some commentary on the research approach.

\section{RESEARCH APPROACH}

The CAPELLA project was a two-year software process improvement (SPI) experiment funded by the European Commission's Esprit initiative (known as ESSI - the European Systems and Software Initiative). Under this scheme, funding was available to software development organisations in order to promote 'best practices'. The organisation concerned was the software development section of the Information Technology Services Division (ITSD) of Salford City Council, a medium-sized UK local government council.

The authors / researchers represented the Information Systems Research Institute (ISRI) at the University of Salford, which, along with the Norwegian Computer Centre (NR) in Oslo, acted as advisors / consultants to the ITSD. The consultancy roles included the provision of:

- advice on CASE implementation

- advice on research design and methodology

- design of the evaluation framework (metrics)

- management of the evaluation phase of the experiment

- data creation, analysis and modelling

- assistance in the dissemination activities, and

- quality review of the various deliverables

Since the researchers were intimately involved in the execution of the experiment alongside managers in the development organisation, then the term 'action research' is most appropriate to describe the approach employed. More specifically, the approach corresponds closely to what Reason and Bradbury (2001, pp. xxv-xxvi) call 'second-person' action research practices, namely face-to-face interpersonal dialogue including the development of communities of enquiry in the learning organisation that focuses on matters of mutual concern. Since the object of our research was 
the 'organisation', then action research, according to Galliers' taxonomy (1992), is a highly appropriate approach. However while this might describe the approach to the CAPELLA project described further in the following section, it does not necessarily apply to this paper that more accurately represents a reflective historical view of some aspects of the project that was formally concluded in April 1999.

The fact that our case study is an historical reflection has both advantages and disadvantages. Advantages include distancing and reflecting over time. Disadvantages are that personal recollections may be flawed and that accounts of the processes may therefore be factually inaccurate. We are not unduly concerned about these; detailed reports and accounts of the project and its evolution have been meticulously kept by those local government officers involved in the project, and additional accounts have been published elsewhere (Wastell et al., 1999a, Wastell et al., 1999b). In any case, we are not so much concerned here with simply trying to recount bald facts. Instead, we are interested to see what we can learn from reflecting on the processes and conduct of the experiment that form part of our own experiences. Historical reflections, as Gummesson (1988) puts it, are about building "hermeneutic bridges" between the past and the present, to enable an increased understanding of the current and future state of the organization. We now proceed to a description of the CAPELLA project.

\section{THE CAPELLA PROJECT}

The CAPELLA project (CAse tools for Process Enhancement in LocaL Authorities) was a two-year Software Process Improvement initiative undertaken between 1997 and 1999, and funded to the sum of $£ 250,000$ under the European Commission's ESSI programme (ESSI Project 23832) as a Process Improvement Experiment (PIE). The principal organisation was the ITSD, with the ISRI at the University of Salford, and the Norwegian Computing Centre in Oslo providing consultancy services. The project had two main aims,

To evaluate and understand the implications of implementing a CASE tool and associated methods, and

To form a wider understanding of the issues and impacts of technological change within an organization.

Put simply, CAPELLA aimed to introduce a CASE tool and new working methods into the department, with the expectation that through this process a number of important benefits might accrue, including: 
- Improved software quality

- Increased developer productivity

- Increased customer involvement

- Reduced development / maintenance costs

- Increased developer job satisfaction

- Improved customer satisfaction

\subsection{The Project plan}

The project plan consisted of three discrete phases; the first of these was concerned with the acquisition, installation and initial training in the use of the CASE tool, selecting those who would make up the initial 'centre of excellence' - that is those who were intended to acquire the technical expertise to subsequently advise others in the next phase of the project and beyond. This was to be initiated through the development of a small pilot project. During this period, a base-line project was also identified as a focus for the main experiment and roll-out of the methodology and tool use. In the first instance, this was to be a 48 person-year project for the Housing Department.

The second phase would be concerned with constructing a development methodology that would accompany the use of the CASE tool. It also concerned the development and capture of various metrics - for example the measurement of (developer) productivity, developer job satisfaction, and attitudinal issues such as customer satisfaction. The third phase was to be the main part of the experiment - the roll-out of the method and tool to wider developer use. This phase also included the requirement to reflect and report on the issues, problems and processes insofar as these had an impact upon the ITSD, and as it affected its customer base and customer perceptions of quality and other matters.

\subsection{Problems encountered}

The project officially began on March $1^{\text {st }} 1997$, but was almost immediately beset by difficulties starting with the loss of the baseline housing project. Over the course of the following year there were a number of additional unforeseen problems that had negative effects on the project including very high staff turnover, low morale, Y2K demands, and other matters. The baseline project was lost early in the process. This had been a 48 person-year integrated housing system featuring rent accounting, property repairs, property allocation and contract management. This was a hard blow, given that the European funding was based upon a proposal that assumed this as a 'given' and an essential basis for underpinning the experiment. A 
much smaller single regeneration budget (SRB) system for the Finance Department was found to replace this, though it never quite met the opportunities promised by the housing system.

A snapshot of the organisation in 1997/98 showed that there was a $27 \%$ rate in staff turnover. This included the ITSD's CAPELLA project leader, a senior member of staff, as well as two of those trained in the CASE tool that formed the centre of excellence, and others. One ironic aspect of this was that having received training in the tool, these developers found they were now more marketable with their newfound skills, and therefore able to command greater remuneration elsewhere than they could in local government.

Low morale was evident in the ITSD. This took several forms, but included a degree of cynicism towards 'just another management fad' as the project was perceived to be by some. Y2K demands were made on the ITSD that involved all staff during 1998. This came down through the larger bureaucracy (from the upper echelons of the Council) and despite the fact that personnel were supposedly ring-fence protected by European funding, they were nevertheless diverted into the pressing demands and preparations for the imminent new millennium. Other problems included the realisation that inadequate standards and methods existed within the ITSD, which had an immature planning and management culture, and that collating metrics was far more problematic than had been hitherto envisaged.

\subsection{Some outcomes}

Work on the implementation process progressed throughout the period March 1997 to April 1999 when the project was formally 'concluded'. The original project proposal was for eighteen months, but some of the difficulties necessitated two requests for short extensions. To put it succinctly and simply, the CASE tool was never fully deployed, not only in terms of the numbers of users - this always remained small - but also in terms of its capabilities and functionality, and it was finally abandoned after some limited use. The developers were unable to incorporate the tool's potential into their working practices for a variety of reasons, not least because of the fact that during this period there was a rapid decline in inhouse bespoke systems in favour of widespread packaged software procurement. Today no development work outside of package configuration and the maintenance of legacy systems takes place.

Despite the failure to adopt the technology, there were also some very positive outcomes; spin-offs from the implementation effort that have bequeathed long-term benefits to the department. These included:

- The establishment of a working 'standards and methods' group 
- Design and implementation of a project planning infrastructure

- Establishment of a peer review process for all present and future projects

- Staff retention measures to combat severe haemorrhaging of skilled staff

- An evolving 'metrics culture'

- Continued and ongoing collaboration with universities

- Establishment of a research and development team

In the face of these contrary results, how should the outcome of this twoyear implementation effort be described? What other lessons can be learned from the case?

\section{DISCUSSION}

There are some similarities between this story and one told by Ginn (1994) where he describes the efforts to implement a computerised timescheduling system into a US aerospace factory. Its purpose was to try to address the difficulties associated with moving aircraft parts in a timely manner during the construction process. Cranes to move aircraft parts need to be booked sometimes many months ahead with a fine degree of accuracy in terms of timing so as not to burden the company with undue overheads. Although the software system failed to become 'accepted, routinized and infused' (Saga \& Zmud, 1994) and was in fact finally abandoned, nevertheless the company found that efficiency in that section was improved by around $15 \%$ as a result of the efforts to implement it.

We are not able to quantify the benefits to the ITSD in quite the same way. However all of the players are agreed that the benefits listed above have been realised. In addition, the ITSD continues to work with universitybased researchers, and has expanded its collaborative efforts further to develop a methodology for handling process reengineering projects, known as SPRINT (Wastell et al., 2001) among other initiatives. Despite this positive outcome, the CASE technology itself was not adopted.

There would appear to be four main implications of the case for our theoretical understanding of technological innovation and its problematics, particularly in the area of software engineering. First, the case shows again (if further demonstration were needed) that methodological innovation is a perilous endeavor, in the sense that the introduction of new methods and tools runs a high risk of encountering resistance and rejection. IT-enabled transformation is generally fraught with the risk of miscarriage and failure, with the mortality rate for re-engineering efforts running as high as 70\% (MacIntosh, 2003; Al-Mashari and Zairi, 1999) and software process innovation is no different. There is a significant body of research on the implementation of new software tools (Hardgrave et al, 2003) which 
evidences substantial resistance and frequent rejection (Orlikowski, 1993; Iivari, 1996). Much greater resistance might be expected for complete methodologies given the scale of re-structuring involved (Hardgrave, 2003). Methodologies are more embracing than individual tools, requiring substantial organizational change and the introduction of new processes and practices, rather than the more localized change entailed by the adoption of an individual tool. The rejection of CASE in ITSD confirms this general picture.

The second general lesson relates to the notion of "failure", specifically to the relative nature of this pejorative concept. Although the developers in the ITSD failed to adopt the CASE tool, is it fair to describe the project as a failure? We think not, as the technical artefact merely reflects a single component in a raft of activities and aims that made up the CAPELLA project, some of which were usefully deployed, others not so. The labels success and failure are attributed too often to initiatives in a naïve and simplistic way. While reports dealing with IS outcomes are relatively commonplace, few deal with these labels in a way that does full justice to the underlying complexity of situations and ideas. One exception is Larsen and Myers (2000), who provide an example of a BPR project in financial services firm in New Zealand. These authors address directly the problematic nature of success and failure, relating how shortly after completion of the project, the accounts of its outcome really depended on who was telling the story. They also suggest that such descriptions are more political declarations than statements of fact, and that success is a "moving target". Another example is found in Irani et al (2001), wherein the initial failure to implement an ERP system in a manufacturing company ultimately came to be seen more sanguinely as paving the way for a successful in-house development. It is clear that a more critical approach is required to the use of the terms success and failure, and that more circumspection is called for in the appellations given to attempts at technological innovation.

The third implication relates to the importance of organizational culture in shaping the trajectory of methodological innovation. Popular models of technological change, embodied in diffusonist formulations such as the Technology Adoption Model (Davis et al, 1989), tend to privilege attributes of the technology in accounts of outcomes. The present work in contrast underlines the importance of organizational culture. IT-enabled change is highly problematic in any organizational domain. The cultural circumstance of large public sector bureaucracies would seem to offer a particularly challenging milieu. They are hardly seen in the public imagination as the standard bearers of change and modernization. Several authors have contended that the failure rate for technologically-based innovation in public organizations is likely to be high (Kock et al, 1996) due to a range of factors: 
greater regulation restricting the range of potential responses, rigid hierarchies and multiple stakeholders for many processes; overlapping initiatives and a rapidly changing policy environment; lack of resources and a staff culture emphasizing the need for consultation and consensual change (MacIntosh, 1997).

Internally, local councils tend to be deeply hierarchical, bureaucratic entities, often steeped in traditional methods and processes that mean changes to practice can be immensely difficult to achieve. Often positions of authority are held by those who have worked their way up through the hierarchy, having joined the organisation as juniors at a relatively young age. Bad practices are thus often preserved and perpetuated as part of the embedded institutionalised culture. We do not suggest that was a problem specific to the ITSD. Nonetheless it was clear that a significant constituency of developers saw the attempts to introduce the CAPELLA project as just another 'management fad' and perhaps therefore not worth making too much of an effort over. Another feature of local government is that there are few other organisations that are quite so explicitly political in nature. All councils are composed of elected members who are typically aligned with specific political parties. Within legal constraints and through controlling committees, they then attempt to implement policies through the departmental structures of the organisation that best reflect the political aspirations of the parties they serve. Due to the relatively transient status of elected members and the often poor understanding they have of the nutsand-bolts detail of departmental service delivery, senior officers (full-time employees) often wield considerable influence and power. The present initiative was certainly supported politically, with a number of elected members and Chief Officers attending the CAPELLA launch. However whether this support cascaded down much beyond the upper hierarchy of the ITSD is not certain. It is possible that the enrolment of politicians by management had been seen as a means of trumpeting their own accomplishments in the eyes of those they strove to impress. Winning European funding by such a small strategic business unit is not common; many large councils often have special units, usually part of their Chief Executive's Departments, set up to exploit European funding opportunities. Making the launch of CAPELLA such a high profile affair also sends out the message, 'we know we have problems, but look, we're serious and we're doing something about it'.

Externally, local government is influenced by the vagaries of central government to a degree far in excess of most other organisations. New legislation and advice means that they have to respond, sometimes at relatively short notice to accommodate expediency and law, such as was the case for example in the introduction of the Community Tax (known as the 
Poll Tax) in 1990. Councils were obliged to build or buy new computerbased systems to deal with this tax. Within two years the then Conservative government under Margaret Thatcher had to abandon this when in open defiance of government policy, many people chose to go to jail rather than pay this highly unpopular tax. There are no published details of the total national cost of the exercise, but it almost certainly ran to hundreds of millions of pounds to UK taxpayers. The 'Council Tax' immediately replaced the Poll Tax, so that yet another major round of software acquisition was demanded of UK councils, and there are many other ongoing legislative requirements that do not attract the same level of public interest. The ITSD are among those that prefer to try and build their own inhouse systems such as Council Tax, rather than purchase from commercial vendors. It is easy to imagine therefore that software developers in local government are not keen to have additional and 'unnecessary initiatives' such as the CAPELLA Project thrust upon them, as it must have appeared to some.

This brings us to our final point. Drawing on Actor Network Theory, we take a general, paradigmatic view of technological change as a dynamic process involving a shifting coalition of "actants", including inanimate elements such as the technology itself. Successful transformation reflects the progressive enrollment of these elements into a stable network that ultimately comes to embody the transformed organizational order. Critical in this process is the first stage, of alignment. This refers to the initial drawing in of "actants" (human and non-human) through problematisation (i.e. establishing that some problem exists and redefining the world accordingly, with the innovation as the key to the solution) and interressement, i.e. attempting to impose on others the new roles and identities implicit in the redefinition. If "successful", interressement leads to the progressive enrolment of actants in a stronger and stronger network of alliances supporting and promulgating the innovation. Finally there is congealment, whereby the innovation consolidates itself as an accepted fact of life, a "black box", as part of the transformed order.

Problematisation is the decisive first step in this transformation. "Success" depended on enrolling key constituencies within ITSD to see the CASE tool as a solution to the key problems perceived as facing the department. Wastell et al (2003) contend that the most important stimulus for organizational change is the perception of a serious threat to the organization. Without such a sense of crisis, inertia will tend to prevail and any mobilization for change will quickly peter out. The work here would seem to confirm this. There was no such sense of crisis strongly aligned with the messianic appearance of CASE. A successful bid for funding had been made, around the need of the organization to improve software quality and 
productivity. Whilst this sufficed to unite three groups of actants (the researchers, senior ITSD management and ESSI) around the rhetorical need for CASE, other key groups (i.e. the software developers who would use the tool) were not enrolled within this problematisation. Amongst these staff groups, CASE certainly figured in some minority problematisations, e.g. the need to advance individual careers, but these would tend to produce organizational fragmentation rather providing a strong force for collective transformation. It is clear that staff groups saw the key threat coming from other sources, Y2K in particular, and the CASE tool came to be seen as marginal within this problematisation. The study thus confirms the contested, dynamic nature of the innovation process and pivotal role of problematisation. The importance of some sense of crisis is attested in which the innovation offers a route to salvation; this is critical in shaping the trajectory and ultimate fate of the innovation. CASE came to suffer the same, unlamented fate as other artefacts, such as the Penny-farthing bicycle, that fail to address relevant problems as defined by influential stakeholder groups (Pinch and Bijker, 1984).

\section{REFERENCES}

Al-Mashari, M. and Zairi, M. (1999), 'BPR implementation process: an analysis of key success and failure factors', in Business Process Management Journal, Vol. 5, pp. 87-112.

Bignell, V. and Fortune, J. (1984), Understanding systems failures, Manchester University press in association with the Open University, Manchester.

Irani, Z., Sharif, A.M. and Love, P.E.D. (2001). Transforming failure into success through organizational learning: an analysis of a manufacturing system', in European Journal of Information Systems, Vol. 10, pp. 55-66.

Bussen, W., and Myers, M., D. (1997), 'Executive information systems failure: a New Zealand case study', in Journal of Information Technology, Vol. 12 (2), pp. 145-153.

Davis, F.D., Bagozzi, R. and Warshaw, P. (1989). User acceptance of technology: a comparison of two theoretical models. Management Science, 35, 982-1003.

Flowers, S. (1996) Software failure: management failure, John Wiley \& Sons, Chichester.

Galliers, R., D. (1992), 'Choosing information systems research approaches' in Galliers, R., D. (Editor), Information Systems Research: issues, methods and practical guidelines, Blackwell Scientific Publications, London, pp. 144-162.

Ginn, M. L. (1994), 'The transisionist as expert consultant: A case study of the installation of a real-time scheduling system in an aerospace factory', In Diffusion, transfer and implementation of information technology (Ed, Levine, L.) Elsevier Science BV, Amsterdam, pp. 179-198.

Gummesson, E., (1988), Qualitative Methods in Management Research, Studentlitteratur, Sweden, Chapter 4.

Hardgrave, B.C., Davis, F. and Riemenschneider, C.K. (2003). 'Investigating determinants of software developers' intentions to follow methodologies', in Journal of Management Information Systems, Vol. 20, pp. 123-151. 
Iivari, J. (1996). 'Why are CASE tools not used?', in Communications of the ACM, Vol. 39, pp. 94-103.

Kautz, K. and T. McMaster (1994), 'The failure to introduce systems development methods: a factor-based analysis', in Levine, L. (Ed), Diffusion, transfer and implementation of information technology, Elsevier Science BV: pp. 275-287.

Kock, N.F., McQueen, R.J. and Baker, M. (1996), 'Re-engineering a public organisation: an analysis of a case of successful failure', International Journal of Public Sector Management, Vol. 9, pp. 42-50.

Larsen, M., A., and Myers, M., D. (2000), 'When success turns to failure: a package-driven business process re-engineering project in the financial services industry' in the Journal of Strategic Information Systems, Vol. 8, pp. 395-417

Lyytinen, K., and Hirschheim, R., (1987), 'Information Systems Failures - a survey and classification of the empirical literature', Oxford Surveys in Information Technology, Vol. 4, pp. 257-309, Oxford University Press.

McMaster, T, Vidgen, R. and Wastell, D. (1997). Technology transfer: diffusion or translation. In: McMaster et al (eds.), Facilitating technology transfer through partnership: learning from practice and research, Ambleside, Chapman \& Hall, 64-75.

McMaster, T., Jones, M., C., \& Wood-Harper, A., T. (1997), 'Designing stakeholder expectations in the implementation of new technology: Can we ever learn our lessons?', in M. Kyng and L. Mathiassen (Eds), Computers and design in context, Cambridge Ma, MIT Press: pp. 239-265.

MacIntosh, R. (2003). 'BPR: alive and well in the public sector', in International Journal of Operations and Production Management, Vol. 23, pp. 327-344.

Reason, P. and Bradbury, H. (Eds.) (2001) Handbook of Action Research: Participative Inquiry and Practice, Sage Publications, London.

Saga, V. L. and Zmud, R. W. (1994), 'The nature and determinants of IT acceptance, routinization and infusion', In Diffusion, transfer and implementation of information technology (Ed, Levine, L.), Elsevier Science BV, Amsterdam, pp. 67-86.

Sauer, C. (1993), Why information systems fail: a case study approach, Henley-on-Thames, Alfred Waller Limited.

Teng, J., T., C. (1998), 'Profiling successful reengineering projects' in Communications of the ACM, Vol. 41 (6), pp. 96-102.

Wastell, D.G, (1996), 'The fetish of technique: methodology as a social defence', in Information Systems Journal, Vol 6(1), pp. 25-40.

Wastell, D., G., Kautz, K., Kawalek, P., and McMaster, T. (1999a), 'Using CASE to Enhance Service Performance in Local Government: The CAPELLA Project', in M. Oivu and P. Kuvaja (eds.), International Conference on Product Focused Software Process Improvement (PROFES'99), VTT, Finland.

Wastell, D., G., Kautz, K., Kawalek, P., and McMaster, T. (1999b), 'Software Process Improvement Using CASE: Lessons from the Front-line', European Software Process Improvement Conference (EUROSPI'99), Pori, Finland

Wastell, D., Kawalek, P. And Newman, M. (2003) Plus ça change: defensive translations and resistance to IT-enabled change in local government. Proceedings of ECIS 2003, Naples, Italy.

Wastell, D.G., Kawalek, P. and Willetts, M. (2001). Designing alignment and improvising change: experiences in the public sector using the SPRINT methodology. In: Smithson et al. (Eds.) Global Cooperation in the New Millennium, Proceedings of ECIS 2001, Bled, Slovenia. 